\title{
Bone morphogenic protein 2 induces Runx2 and osteopontin expression in human aortic valve interstitial cells: Role of Smad1 and extracellular signal-regulated kinase 1/2
}

\author{
Xiaoping Yang, PhD, Xianzhong Meng, MD, PhD, Xin Su, MD, David C. Mauchley, MD, Lihua Ao, BS, \\ Joseph C. Cleveland, Jr, MD, and David A. Fullerton, MD
}

\begin{abstract}
Objective: Bone morphogenic protein 2 is found in calcified areas of stenotic aortic valves, and prolonged stimulation of aortic valve interstitial cells with bone morphogenic protein 2 results in increased expression of alkaline phosphatase, indicating a mechanistic role for bone morphogenic protein 2 in aortic valve calcification. The purposes of this study were to assess the effect of bone morphogenic protein 2 on the expression of the osteogenic factors Runx 2 and osteopontin in human aortic valve interstitial cells and to determine the signaling mechanisms that mediate the expression of these early osteogenic factors.
\end{abstract}

\begin{abstract}
Methods: Interstitial cells were isolated from normal and stenotic human aortic valve leaflets, and cellular bone morphogenic protein 2 levels were analyzed by means of immunoblotting. Cultured interstitial cells from normal aortic valves were stimulated with bone morphogenic protein 2 to determine its effect on cellular Runx2 and osteopontin levels.
\end{abstract}

Results: Interstitial cells from stenotic aortic valves express greater levels of bone morphogenic protein 2 than cells from normal valves. Stimulation of human aortic valve interstitial cells with bone morphogenic protein 2 induced marked increases in Runx 2 and osteopontin levels at 48 hours. The changes in Runx 2 and osteopontin levels were preceded by phosphorylation of Smad1 and extracellular signal-regulated kinase 1/2 but not p38 mitogen-activated protein kinase. Silencing Smad1 reduced Runx2 and osteopontin levels, whereas inhibition of extracellular signal-regulated kinase 1/2 reduced osteopontin expression without an influence on Runx2 expression.

Conclusions: Interstitial cells of stenotic human aortic valves are characterized by increased bone morphogenic protein 2 levels. A short period of exposure of human aortic valve interstitial cells to bone morphogenic protein 2 induces the expression of Runx 2 and osteopontin. The extracellular signal-regulated kinase 1/2 pathway modulates bone morphogenic protein 2-induced osteopontin expression, and the Smad1 pathway plays a role in regulating the expression of both Runx 2 and osteopontin induced by bone morphogenic protein 2 .

\section{Supplemental material is available online.}

Calcific aortic stenosis is the third leading cardiovascular disease behind only hypertension and coronary artery disease. ${ }^{1}$ It is found in approximately $3 \%$ of the population older than 65 years and is the most common indication for heart valve replacement surgery. ${ }^{2}$ Long considered a disease of passive degeneration, evidence has emerged that aortic stenosis might result from an active disease process. How-

From the Division of Cardiothoracic Surgery, Department of Surgery, University of Colorado Denver, Aurora, Colo.

Supported in part by American Heart Association grant $0850148 \mathrm{Z}$.

Received for publication March 16, 2009; revisions received May 20, 2009; accepted

for publication June 20, 2009; available ahead of print Aug 7, 2009.

Address for reprints: Xianzhong Meng, MD, PhD, Department of Surgery, Box C-320,

12700 E 19th Ave, Aurora, CO 80045 (E-mail: Xianzhong.meng@ucdenver.edu).

J Thorac Cardiovasc Surg 2009;138:1008-15

$0022-5223 / \$ 36.00$

Copyright (c) 2009 by The American Association for Thoracic Surgery

doi:10.1016/j.jtcvs.2009.06.024 ever, the mechanisms responsible for the development of aortic stenosis remain unclear.

Insight into the pathogenesis of calcific aortic stenosis has been gained from the examination of aortic valve leaflets explanted at aortic valve replacement. Explanted calcified valve leaflets demonstrate morphologic features resembling osteogenic bone formation, as well as the expression of proteins associated with bone formation. ${ }^{3}$ The fact that diseased aortic valve leaflets have such a histologic appearance suggests that calcific aortic stenosis might result from a process similar to that of active bone formation. This further implies that bone-forming cells (osteoblast-like cells) might be responsible for the calcification. Although the origin of such bone-forming cells is unclear, aortic valve interstitial cells (ICs) have been shown to have the ability to become osteoblast-like cells. ${ }^{4}$

Bone morphogenic proteins are osteogenic growth factors that belong to the transforming growth factor $\beta$ superfamily. These osteogenic growth factors are the principal inducers of osteoblast differentiation and bone formation. They act through both autocrine and paracrine mechanisms, and the 

Abbreviations and Acronyms
BMP-2 = bone morphogenic protein 2
ERK = extracellular signal-regulated kinase
IC $=$ interstitial cell
MAPK $=$ mitogen-activated protein kinase
PBS = phosphate-buffered saline
siRNA $=$ small interfering RNA

paracrine effects are particularly important in osteogenesis. ${ }^{5}$ Bone morphogenic protein 2 (BMP-2) has been found to play critical roles in vascular calcification. ${ }^{6}$ This osteogenic growth factor has been identified in calcified tissue of stenotic aortic valves. ${ }^{7,8}$ Likewise, the bone-forming protein osteopontin has been found in calcified aortic valve leaflets. ${ }^{2}$ However, whether aortic valve ICs can be stimulated to produce osteopontin is unclear. After stimulation with BMP-2, the production of bone-forming proteins, such as osteopontin, requires the production of the transcription factor Runx 2 in certain cell types. Hence in the present study the production of osteopontin and Runx 2 after stimulation with BMP-2 was studied.

Although ICs appear to play an important role in aortic valve calcification, ${ }^{9}$ it is unclear whether BMP-2 expression in ICs is altered in stenotic aortic valves. In a recent study ${ }^{10}$ we isolated ICs from the noncalcified tissue of stenotic aortic valves and found that they are phenotypically different from ICs of normal valves. Analysis of BMP-2 expression in ICs of stenotic aortic valves might provide insights into the pathophysiology of aortic valve stenosis and calcification.

Previous studies by our group and others have found that stimulation of human aortic valve ICs for 2 or 3 weeks with BMP-2 upregulates the expression of alkaline phosphatase. ${ }^{4,10}$ In C2C12 cells, BMP-2 activates the Runx2 pathway through Smad and p38 mitogen-activated protein kinase (MAPK). ${ }^{11-13}$ The roles of the Smad and p38 MAPK pathways in mediating the effect of BMP-2 on human aortic valve ICs remain unclear. Further determination of the effect of BMP-2 on human aortic valve IC osteogenic response and the underlying signaling mechanisms is important to elucidate the role of BMP-2 in the pathogenesis of aortic valve calcification and stenosis.

In the present study we sought to determine (1) whether ICs of stenotic human aortic valves express higher levels of BMP-2, (2) the effect of BMP-2 on the expression of the early osteogenic factors Runx2 and osteopontin in human aortic valve ICs, and (3) the signaling mechanisms that mediate the effect of BMP-2 on the expression of these early osteogenic factors.

The results of the present study offer mechanistic insight into the pathogenesis of calcific aortic stenosis. The ICs of stenotic human aortic valves were found to be characterized by increased BMP-2 levels. Exposure of normal human aortic valve ICs to BMP-2 induces the expression of Runx 2 and osteopontin. The extracellular signal-regulated kinase (ERK) 1/2 pathway modulates BMP-2-induced osteopontin expression, and the Smad1 pathway plays a role in regulating the expression of both Runx 2 and osteopontin induced by BMP-2.

\section{MATERIALS AND METHODS \\ Chemicals and Reagents}

M199 medium and human serum albumin were purchased from Lonza (Walkersville, Md). Rabbit polyclonal antibody against human BMP-2 was obtained from ProSci (Poway, Calif). Rabbit polyclonal antibody against Runx2 was purchased from Novus Biologicals (Littleton, Colo). Rabbit polyclonal antibody against osteopontin was purchased from Abcam (Cambridge, Mass). Antibodies against phosphorylated and nonphosphorylated Smad1, ERK1/2, and p38 MAPK were purchased from Cell Signaling Technology (Danvers, Mass). Recombinant human BMP-2 was obtained from R\&D Systems (Minneapolis, Minn). PD98059 was purchased from Calbiochem (San Diego, Calif). Smad1 small interfering RNA (siRNA) and DharmaFECT1 transfection reagent were purchased from Dharmacon (Lafayette, Colo). Opti-MEM I medium was purchased from Invitrogen (Carlsbad, Calif). Collagenase and other reagents were purchased from Sigma (St Louis, Mo).

\section{Cell Isolation and Culture}

Normal aortic valves were collected from 5 explanted hearts of heart transplant recipients with cardiomyopathy ( 3 male and 2 female patients 49-65 years old), and stenotic aortic valves were collected from 5 patients who underwent valve replacement surgery for calcific aortic stenosis ( 2 male and 3 female patients 53-72 years old). All valves were tricuspid. This study was approved by the Colorado Multiple Institutional Review Board, and all patients provided written informed consent. On gross examination, normal valve leaflets were thin, and their surfaces were smooth. Microscopic examination of hematoxylin and eosin-stained cryosections confirmed that no leukocytes had infiltrated the leaflets. All stenotic valves were thick and exhibited overt calcification. Noncalcific tissue was used for cell isolation.

Small portions of each valve were either processed into tissue homogenate, frozen in OCT for tissue staining, or used for IC isolation. ICs were isolated by means of collagenase digestion, as previously described, ${ }^{10,14}$ and cultured in growth medium (M199 medium with penicillin G, streptomycin, amphotericin B, and 10\% fetal bovine serum) in an incubator supplied with $5 \% \mathrm{CO}_{2}$. Cells of passage 1 were used for analysis of cellular BMP-2 levels. Cells of passages 3 to 5 were grown to approximately $90 \%$ confluence and treated with recombinant human BMP-2 (100 ng/mL) for a varied period of time to examine the osteogenic response.

\section{Immunohistochemistry}

Immunohistochemical detection of BMP-2 protein in aortic valve leaflets was performed by using the biotin-linked peroxidase technique. Cryosections $(5 \mu \mathrm{m})$ were prepared and dried at room temperature for 2 hours. Sections were fixed with $4 \%$ paraformaldehyde in phosphate-buffered saline (PBS) at room temperature for 10 minutes and then washed with PBS. Unless indicated, all incubations were performed at room temperature. Endogenous peroxidase activities were quenched by incubating sections with $1 \% \mathrm{H}_{2} \mathrm{O}_{2}$ in methanol for 10 minutes. Sections were incubated for 30 minutes with $10 \%$ goat serum in PBS to block nonspecific binding sites. Sections were then incubated for 90 minutes with rabbit 


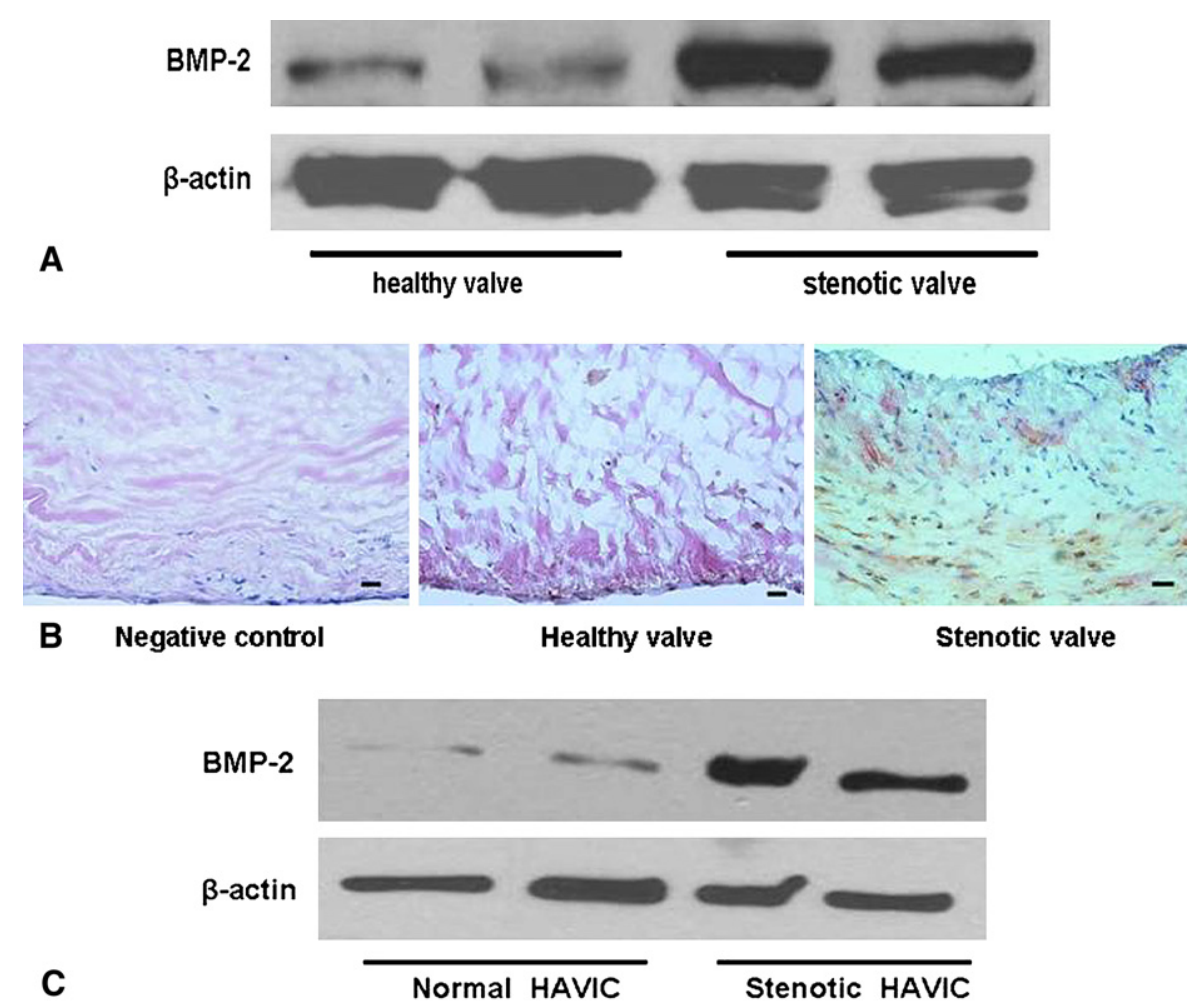

FIGURE 1. Stenotic aortic valves express higher levels of bone morphogenic protein 2 (BMP-2). A representative immunoblot of aortic valve tissue homogenates (A) and immunohistochemical staining of aortic valve leaflets (B) show that stenotic human aortic valves produced higher levels of BMP-2 than normal valves. BMP-2 is represented by the brown color in the immunohistochemistry images. The black bar in the lower left corner of each image represents $20 \mu \mathrm{m}$. Aortic valve interstitial cells isolated from stenotic valves also have higher levels of BMP-2 than cells isolated from normal valves (C). HAVIC, Human aortic valve interstitial cells.

polyclonal antibody against BMP-2 $(5 \mu \mathrm{g} / \mathrm{mL}$ in PBS containing $10 \%$ goat serum) followed by a 60 -minute incubation with biotinylated goat anti-rabbit $\operatorname{IgG}(1: 200$ dilution with PBS containing 10\% goat serum) from the Rabbit ABC Staining System (Santa Cruz Biotechnology). Subsequently, sections were washed 3 times with PBS and incubated with avidin-biotin-peroxidase complex (1:50 dilution with PBS) for 30 minutes. After thorough washes with PBS, color development was carried out with peroxidase substrate $\left(0.03 \% \mathrm{H}_{2} \mathrm{O}_{2}\right.$ and $0.05 \%$ diaminobenzidine in double-distilled $\mathrm{H}_{2} \mathrm{O}$ ). Sections were counterstained with hematoxylin and eosin.

\section{Silencing Smad1}

A transfection mixture of Smad1 siRNA (60 or $90 \mathrm{nmol} / \mathrm{L}$ ) and DharmaFECT1 transfection reagent $(0.5 \mu \mathrm{L} / \mathrm{mL})$ was made in Opti-MEM I reduced serum medium. ICs were incubated with the transfection mixture in complete medium for 48 hours, which we found to be the optimal transfection time. Cells were incubated in growth medium for 24 hours after transfection and then stimulated with BMP-2. Controls were treated with scrambled siRNA $(90 \mathrm{nmol} / \mathrm{L})$ and DharmaFECT1 transfection reagent. Table E1 lists the sequences of sense and antisense siRNA nucleotides.

\section{Immunoblotting}

Cell lysates were separated on $4 \%$ to $20 \%$ minigels and transferred onto nitrocellulose membranes (Bio-Rad Laboratories, Hercules, Calif). After blocking with $5 \%$ fat-free milk solution, the protein of interest was detected by using a primary antibody and a corresponding peroxidase-linked secondary antibody (Cell Signaling Technology) diluted 1:5000. Blots were devel- oped with ECL reagent and exposed on x-ray film. Films were scanned, and Image $\mathbf{J}$ software was used to quantitate band density.

\section{Statistical Analysis}

Densitometric data from more than 2 groups were compared and analyzed by means of analysis of variance with the post hoc Fisher's test, and single comparisons were made with the unpaired $t$ test. Statistical significance was accepted within a $95 \%$ confidence limit.

\section{RESULTS}

\section{Stenotic Aortic Valves and ICs From Stenotic Valves Express Higher Levels of BMP-2}

We have previously reported the BMP-2 protein levels are higher in cells of stenotic aortic valves than in normal valves after stimulation of Toll-like receptors. ${ }^{10}$ In the present study we used immunoblotting to examine BMP-2 protein levels in ICs isolated from normal and stenotic aortic valves. We confirmed that BMP-2 protein levels were markedly higher in tissue from stenotic valves (Figure 1, $A$ and $B$ ), as well as in cells isolated from stenotic aortic valves (Figure 1,C). BMP-2 localizes to the valvular ICs on immunohistochemical staining and appears to have a cytoplasmic distribution (Figure $1, B$ ). Therefore ICs constitute an important 


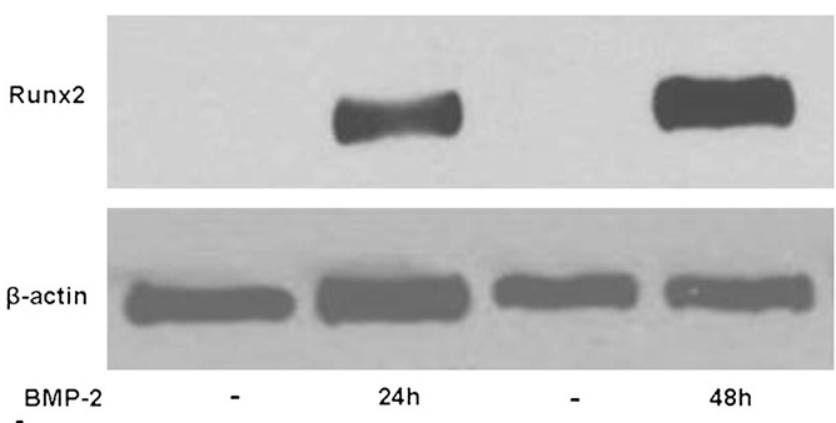

A

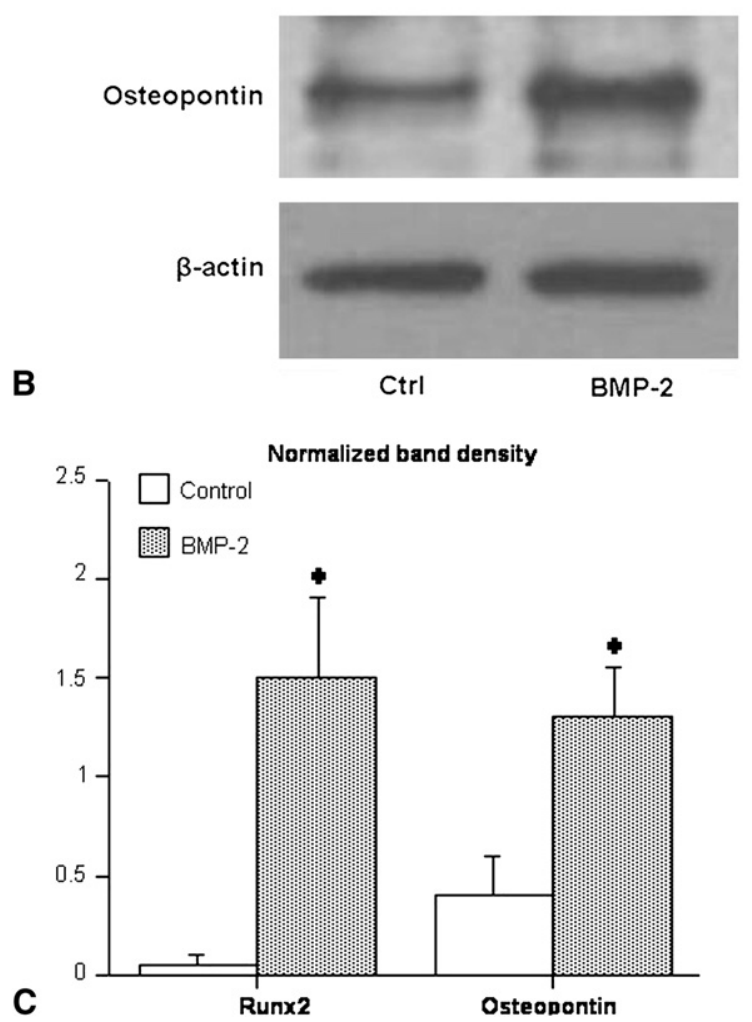

FIGURE 2. Bone morphogenic protein $2(B M P-2)$ induces Runx 2 and osteopontin expression in interstitial cells. A, Representative immunoblot shows that BMP-2 stimulation induced Runx 2 expression at 24 hours, and cellular Runx2 level increased further with 48 hours of stimulation. B, Osteopontin levels increased in interstitial cells stimulated with BMP-2 for 48 hours in comparison with those seen in control ( $\mathrm{Ctrl}$; untreated) cells. C, Densitometric data show that both Runx 2 and osteopontin levels are significantly increased after 48 hours of BMP-2 stimulation. Data are expressed as means \pm standard errors $(\mathrm{n}=3)$. $* P<.05$ versus control.

cell type in the expression of BMP-2 in the stenotic aortic valve.

\section{BMP-2 Upregulates Runx2 and Osteopontin Protein Levels in Human Aortic Valve ICs}

Previous studies by our group and others have shown that prolonged stimulation of human aortic valve ICs with BMP-2 upregulates the protein level of alkaline
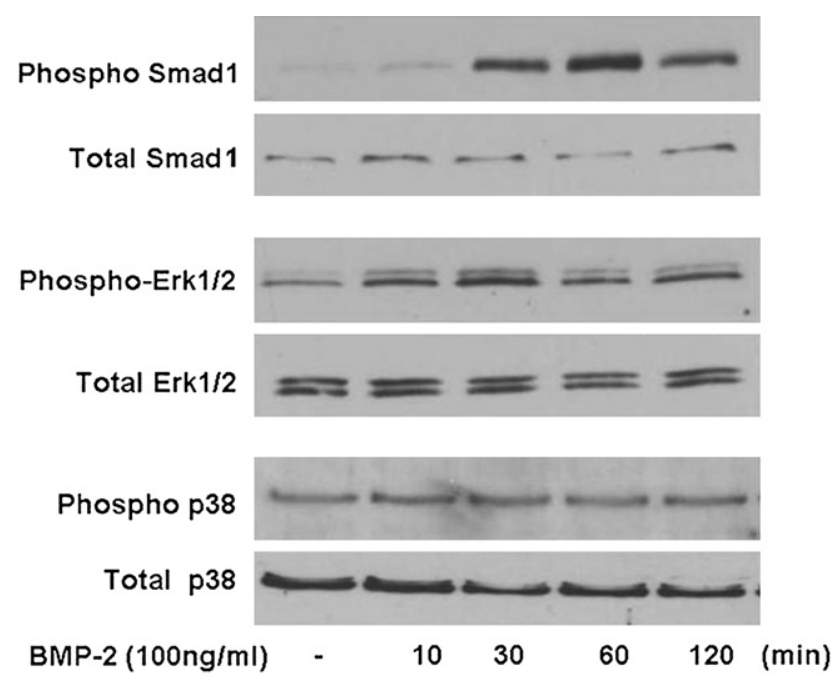

FIGURE 3. Bone morphogenic protein $2(B M P-2)$ induces Smad1 and extracellular signal-regulated kinase $1 / 2(E R K 1 / 2)$ phosphorylation in interstitial cells. Representative immunoblots of 2 separate experiments show that BMP-2 induced rapid phosphorylation of Smad1 and ERK1/ 2. Peak Smad1 phosphorylation was at 60 minutes, and peak ERK1/2 phosphorylation was at 30 minutes. However, BMP-2 stimulation failed to induce p38 mitogen-activated protein kinase phosphorylation in interstitial cells.

phosphatase, a marker of osteogenic phenotypic change. ${ }^{4,10}$ To further study the effect of BMP-2 on the osteogenic response in human aortic valve ICs, we stimulated cells from normal aortic valves with BMP-2 for 24 to 48 hours and determined its effect on cellular Runx2 and osteopontin levels. Stimulation with BMP-2 increased Runx2 (Figure 2, A) and osteopontin (Figure 2, $B)$ levels. At 48 hours, cellular Runx2 levels increased 29-fold and osteopontin levels increased 2.3-fold (Figure 2, C). Thus the results demonstrate that BMP-2 upregulates the expression of early osteogenic factors in human aortic valve ICs.

\section{BMP-2 Activates the Smad1 and ERK1/2 Pathways, but Not the p38 MAPK Pathway, in Human Aortic Valve ICs}

To examine the signaling pathways involved in the BMP2-induced osteogenic response, we treated the cells for 10 minutes to 2 hours and analyzed phosphorylation of Smad1, ERK1/2, and p38 MAPK. Phosphorylated Smad1 was detected at 30 minutes and reached a peak after $60 \mathrm{~min}$ utes (Figure 3). ERK1/2 phosphorylation reached a maximum at 30 minutes (Figure 3). However, p38 MAPK phosphorylation was not detected during the 2-hour stimulation with BMP-2 (Figure 3). Thus BMP-2 activates the Smad1 and ERK1/2 pathways, but not the p38 MAPK pathway, in human aortic valve ICs. 

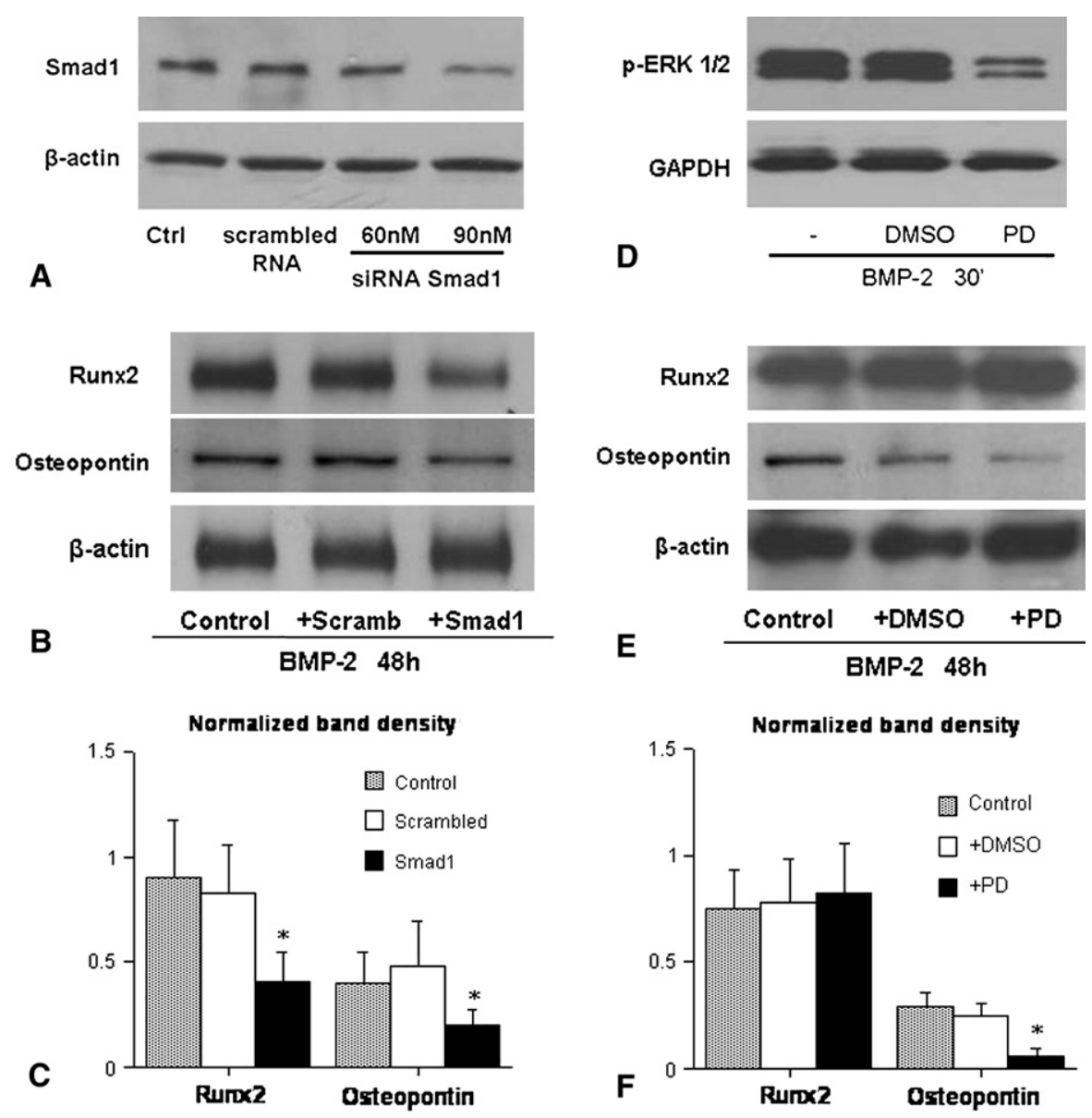

FIGURE 4. Silencing Smad1 reduces the expression of both Runx2 and osteopontin after bone morphogenic protein $2(B M P-2)$ stimulation, whereas inhibiting extracellular signal-regulated kinase 1/2 (ERK1/2) phosphorylation only reduces expression of osteopontin. A, Treatment with Smad1 small interfering RNA (siRNA) at concentrations of 60 and $90 \mathrm{nmol} / \mathrm{L}$ reduced cellular Smad1 levels by $25 \%$ and $51 \%$, respectively. B, Pretreatment of cells with Smad 1 siRNA at $90 \mathrm{nmol} / \mathrm{L}$ reduced Runx2 and osteopontin levels after 48 hours of BMP-2 stimulation. C, Densitometric data show that silencing Smad 1 reduced BMP-2-induced expression of Runx 2 and osteopontin by $54 \%$ and $46 \%$, respectively. Data are expressed as means \pm standard errors $(\mathrm{n}=3$ ). $* P<.05$ versus untreated control and scrambled RNA control. D, Treatment with PD $98059(P D ; 25 \mu \mathrm{mol} / \mathrm{L})$ markedly reduced ERK1/2 phosphorylation induced by BMP-2, whereas vehicle dimethyl sulfoxide (DMSO) had no effect. GAPDH, Glyceraldehyde-3-phosphate dehydrogenase. E, Inhibition of ERK1/2 had no effect on BMP-2-induced Runx2 expression. In contrast, it reduced cellular osteopontin level after BMP-2 stimulation. F, Densitometric data show that inhibition of ERK1/2 reduced BMP-2-induced osteopontin expression by $76 \%$. Data are expressed as means \pm standard errors $(\mathrm{n}=3$ ). $* P<.05$ versus untreated control and DMSO control.

\section{Smad1 and ERK1/2 Pathways Differentially Regulate BMP-2-Induced Runx2 and Osteopontin Expression}

Because BMP-2 stimulation activates the Smad1 and ERK1/2 pathways in human aortic valve ICs, we determined their roles in modulating the expression of Runx2 and osteopontin induced by BMP-2. We used siRNA to silence Smad1. As shown in Figure 4, A, treatment of cells with Smad1 siRNA reduced Smad1 protein levels by $25 \%$ (60 nmol/L siRNA) and 51\% (90 nmol/L siRNA). Using Smad 1 siRNA at a concentration of $90 \mathrm{nmol} / \mathrm{L}$, we found that silencing Smad1 reduced the expression of both Runx 2 and osteopontin after BMP-2 stimulation by $54 \%$ and $46 \%$, respectively (Figure $4, B$ and $C$ ). We used the specific ERK1/2 inhibitor PD 98059 to determine whether the ERK1/2 pathway is involved in the BMP-2-induced expression of Runx 2 and osteopontin. Figure $4, D$, shows that PD 98059 at $25 \mu \mathrm{mol} / \mathrm{L}$ markedly reduced BMP2-induced ERK1/2 phosphorylation. Interestingly, PD 98059 reduced osteopontin expression by $76 \%$ but had no effect on Runx2 expression induced by BMP-2 (Figure $4, E$ and $F$ ). These results demonstrate that the Smad1 pathway plays a role in regulating the expression of both Runx2 and osteopontin induced by BMP-2, whereas the ERK1/2 pathway modulates BMP-2-induced osteopontin expression. 


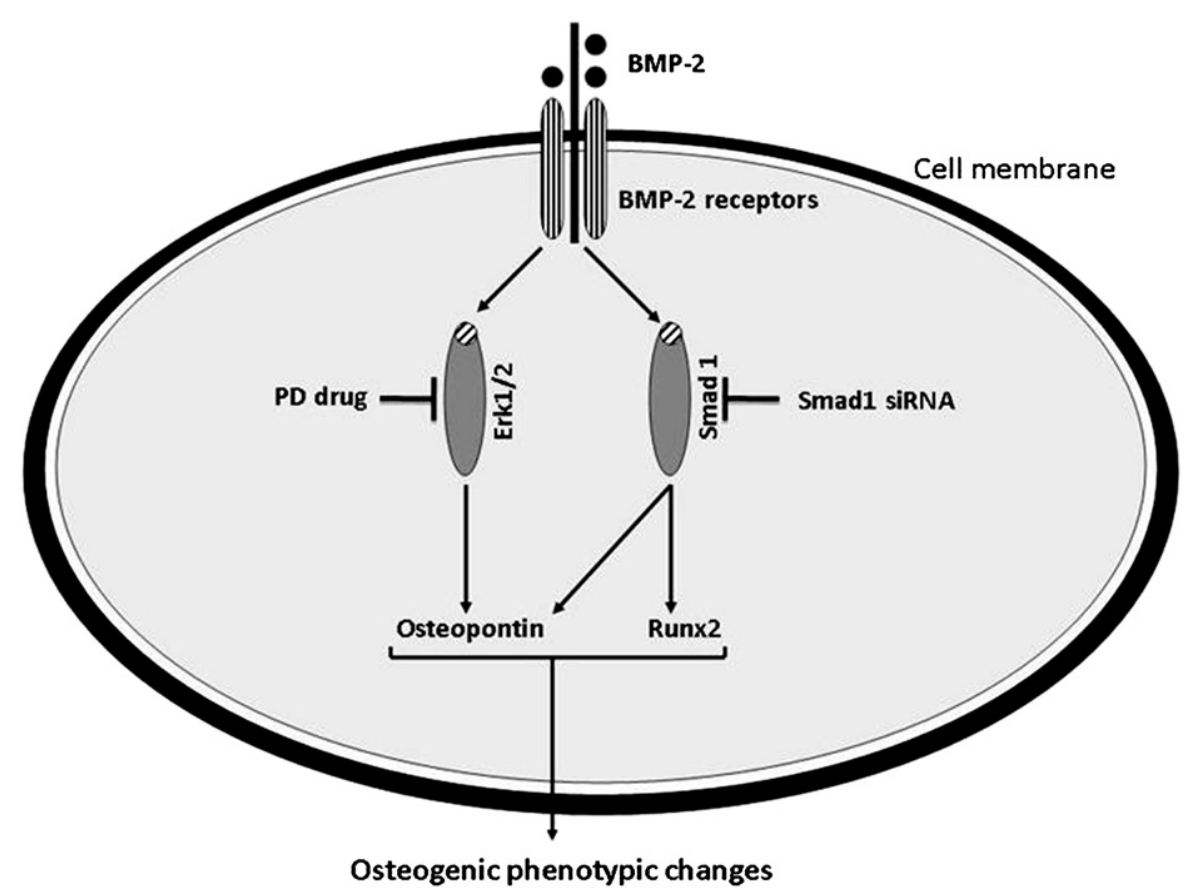

FIGURE 5. Schematic illustration of the role of bone morphogenic protein $2(B M P-2)$ in the development of an osteogenic phenotype in human aortic valve interstitial cells. PD, PD 98059; Erk1/2, extracellular signal-regulated kinase 1/2; siRNA, small interfering RNA.

\section{DISCUSSION}

The results of the present study demonstrate that ICs of stenotic aortic valves express higher levels of BMP-2. Furthermore, the results demonstrate that BMP-2 induces the osteogenic biomarkers Runx2 and osteopontin in human aortic valve ICs in vitro. The Smad1 pathway is required for both Runx2 and osteopontin expression, whereas the ERK1/2 pathway mediates osteopontin expression. These data suggest that the actions of BMP-2 in ICs might play a mechanistic role in the pathogenesis of calcific aortic stenosis.

Several studies have found BMP-2 accumulation in calcification foci of stenotic aortic valves. ${ }^{7,8}$ The results of the present study expand on these observations and demonstrate that ICs isolated from stenotic valves express significantly higher levels of BMP-2, demonstrating that ICs constitute an important source of BMP-2 in diseased aortic valves. It is possible that BMP-2 from aortic valve ICs exerts a paracrine effect on neighboring ICs and other cells to promote calcification. The mechanism underlying the upregulated BMP-2 expression remains unknown from this study; however, proinflammatory cytokines, such as tumor necrosis factor $\alpha$ and interleukin $1 \beta$, have been found to induce BMP-2 expression in human aortic valve ICs in vitro. ${ }^{15,16}$ Work from our laboratory has previously demonstrated that stimulation of Toll-like receptors 2 and 4, 2 important innate immune receptors involved in the mediation of mechanisms of inflammation, also upregu- lates BMP-2 expression in human aortic valve ICs at both the mRNA and protein levels. ${ }^{14,17}$ It is likely that proinflammatory mechanisms are involved in the upregulation of BMP-2 expression in ICs of stenotic aortic valves.

Previous studies have demonstrated that prolonged stimulation of human aortic valve ICs with BMP-2 induces an osteogenic phenotypic change characterized by overexpression of alkaline phosphatase, indicating a critical role for this osteogenic growth factor in valvular cell calcification. ${ }^{4,10}$ Indeed, work from our laboratory has demonstrated that the bone morphogenic protein antagonist noggin reduces alkaline phosphatase expression and calcific nodule formation in human aortic valve ICs exposed to proinflammatory stimuli. ${ }^{10}$ These findings highlight the potential role of BMP-2 in aortic valve stenosis and calcification. In the present study, we determined the effect of BMP-2 on the expression of the early osteogenic mediators Runx2 and osteopontin in human aortic valve ICs. The results of the present study demonstrate that stimulation of cells in vitro with BMP-2 for 48 hours increased cellular Runx2 and osteopontin levels by 29- and 2.3-fold, respectively. The potent effect of BMP-2 on Runx 2 expression is particularly interesting because Runx 2 is an important transcription factor in regulating fibroblast differentiation and bone formation. ${ }^{18}$ Higher Runx2 levels might promote osteoblast-like phenotypic changes in human aortic valve ICs. Further study is necessary to determine the role of 
Runx2 in BMP-2-mediated aortic valve IC expression of alkaline phosphatase and calcification.

The intracellular BMP-2 signaling pathway has been extensively studied in osteoblasts and chondroblasts. ${ }^{19}$ BMP2 has been shown to activate Runx2 through Smad1 and p38 MAPK in $\mathrm{C} 2 \mathrm{C} 12$ and other mesenchymal cells. ${ }^{11-13}$ The results of the present study demonstrate that in human aortic valve ICs BMP-2 induces rapid phosphorylation of Smad1 and ERK1/2 but has no effect on p38 MAPK phosphorylation, indicating that it activates the Smad1 and ERK1/2 pathways to upregulate the expression of Runx2 and osteopontin. We used siRNA and a specific inhibitor to determine the roles of Smad1 and ERK1/2 in the osteogenic response to BMP-2. Silencing Smad1 reduced BMP-2-induced expression of Runx 2 and osteopontin, indicating that the Smad1 pathway is required for the induction of the osteogenic response by BMP-2 in human aortic valve ICs. However, inhibition of ERK1/2 only reduced osteopontin expression. It is noteworthy that inhibition of ERK $1 / 2$ reduced cellular osteopontin levels by $76 \%$, indicating the ERK1/2 pathway has a major role in regulating BMP-2-induced osteopontin expression. It appears that the Smad1 pathway selectively regulates Runx2 expression, whereas the Smad1 and ERK1/2 signaling pathways have an overlapping function in the regulation of osteopontin expression. Signaling pathways might communicate or cross-talk in regulation of cellular response to stimuli. The Smad1 pathway might interact with the ERK1/2 pathway. ${ }^{20}$ Further study is needed to determine whether Smad1 is involved in ERK1/2 activation in the signaling mechanisms underlying the osteogenic response to BMP-2 in human aortic valve ICs.

Calcific aortic stenosis has long been considered a degenerative disease, one in which calcium passively accumulates on the leaflets of the valve. However, some investigators have suggested that the pathogenesis of calcific aortic stenosis might be an active disease process involving osteogenesis. ${ }^{2}$ Another contributing mechanism might also be that of ectopic calcification of the aortic valve. Ectopic tissue calcification is a process by which hydroxyapatite crystals are deposited in tissue; hydroxyapatite crystals can be found in the leaflets of aortic stenosis. Although the results of the present study are consistent with the possibility that mechanisms of osteogenesis might contribute to the pathogenesis of aortic stenosis, speculation as to the type of osteogenesis is not possible.

The results of the present study are consistent with our global hypothesis that aortic valve ICs might contribute to the pathogenesis of calcific stenosis by changing their phenotype and behaving as bone forming-like cells. Future work will focus on examination of the possible links between mechanisms of inflammation and mechanisms of osteogenesis in the pathogenesis of aortic stenosis. A limitation of the present study, however, is the fact that it only studied isolated aortic ICs from healthy valves. Be- fore studying the mechanisms of stenosis with cells from stenotic valves, we believed that it was first important to gain an understanding of these mechanisms in normal valve cells. Future studies with ICs from stenotic valves will be undertaken once the intracellular signal transduction pathways of ICs from healthy valve cells are better understood. Another limitation is that we studied isolated ICs in culture. All such studies are limited by the possibility that the behavior of cells in vitro might differ from that in vivo.

In conclusion, the present study found that ICs of stenotic human aortic valves are characterized by increased BMP-2 levels. A short period of exposure of human aortic valve ICs to BMP-2 induces the expression of Runx2 and osteopontin. The ERK1/2 pathway modulates BMP-2-induced osteopontin expression, and the Smad1 pathway plays a role in regulating the expression of both Runx2 and osteopontin induced by BMP-2 (Figure 5). The results of the present study offer mechanistic insight into the pathogenesis of calcific aortic valve stenosis.

We thank Yimu Yang, MD, PhD, for his technical assistance with immunohistochemical staining.

\section{References}

1. Akat K, Borggrefe M, Kaden JJ. Aortic valve calcification: basic science to clinical practice. Heart. 2009;95:616-23.

2. Rajamannan NM, Bonow RO, Rahimtoola SH. Calcific aortic stenosis: an update. Nat Clin Pract Cardiovasc Med. 2007;4:254-62.

3. Leskela HV, Satta J, Oiva J, Eriksen H, Juha R, Korkiamaki P, et al. Calcification and cellularity in human aortic heart valve tissue determine the differentiation of bone-marrow-derived cells. J Mol Cell Cardiol. 2006;41:642-9.

4. Osman L, Yacoub MH, Latif N, Amrani M, Chester AH. Role of human valve interstitial cells in valve calcification and their response to atorvastatin. Circulation. 2006;114(suppl):I547-52.

5. Sykaras N, Opperman LA. Bone morphogenetic proteins (BMPs): how do they function and what can they offer the clinician? J Oral Sci. 2003;45:57-73.

6. Shao JS, Cai J, Towler DA. Molecular mechanisms of vascular calcification: lessons learned from the aorta. Arterioscler Thromb Vasc Biol. 2006;26: 1423-30.

7. Kaden JJ, Bickelhaupt S, Grobholz R, Vahl CF, Hagl S, Brueckmann M, et al. Expression of bone sialoprotein and bone morphogenetic protein-2 in calcific aortic stenosis. J Heart Valve Dis. 2004;13:560-6.

8. Mohler ER 3rd, Gannon F, Reynolds C, Zimmerman R, Keane MG, Kaplan FS. Bone formation and inflammation in cardiac valves. Circulation. 2001;103: 1522-8.

9. Liu AC, Joag VR, Gotlieb AI. The emerging role of valve interstitial cell phenotypes in regulating heart valve pathobiology. Am J Pathol. 2007;171: 1407-18.

10. Yang X, Fullerton DA, Su X, Ao L, Cleveland JC, Meng X. Pro-osteogenic phenotype of human aortic valve interstitial cells is associated with higher levels of Toll-like receptors 2 and 4 and enhanced expression of bone morphogenetic protein 2. J Am Coll Cardiol. 2009;53:491-500.

11. Kim HJ, Park HD, Kim JH, Cho JY, Choi JY, Kim JK, et al. Establishment and characterization of a stable cell line to evaluate cellular Runx2 activity. J Cell Biochem. 2004;91:1239-47.

12. Lee KS, Hong SH, Bae SC. Both the Smad and p38 MAPK pathways play a crucial role in Runx 2 expression following induction by transforming growth factorbeta and bone morphogenetic protein. Oncogene. 2002;21:7156-63.

13. Zamurovic N, Cappellen D, Rohner D, Susa M. Coordinated activation of notch, Wnt, and transforming growth factor-beta signaling pathways in bone morphogenic protein 2-induced osteogenesis. Notch target gene Heyl inhibits mineralization and Runx2 transcriptional activity. J Biol Chem. 2004;279: 37704-15. 
14. Meng X, Ao L, Song Y, Babu A, Yang X, Wang M, et al. Expression of functional Toll-like receptors 2 and 4 in human aortic valve interstitial cells: potential roles in aortic valve inflammation and stenosis. Am J Physiol Cell Physiol. 2008;294: C29-35.

15. Kaden JJ, Dempfle CE, Grobholz R, Tran HT, Kilic R, Sarikoc A, et al. Interleukin-1 beta promotes matrix metalloproteinase expression and cell proliferation in calcific aortic valve stenosis. Atherosclerosis. 2003;170:205-11.

16. Kaden JJ, Kilic R, Sarikoc A, Hagl S, Lang S, Hoffmann U, et al. Tumor necrosis factor alpha promotes an osteoblast-like phenotype in human aortic valve myofibroblasts: a potential regulatory mechanism of valvular calcification. Int $J \mathrm{Mol}$ Med. 2005;16:869-72.
17. Babu AN, Meng X, Zou N, Yang X, Wang M, Song Y, et al. Lipopolysaccharide stimulation of human aortic valve interstitial cells activates inflammation and osteogenesis. Ann Thorac Surg. 2008;86:71-6.

18. Hassan MQ, Tare RS, Lee SH, Mandeville M, Morasso MI, Javed A, et al. BMP2 commitment to the osteogenic lineage involves activation of Runx 2 by DLX 3 and a homeodomain transcriptional network. J Biol Chem. 2006;281:40515-26.

19. Chen D, Zhao M, Mundy GR. Bone morphogenetic proteins. Growth Factors. 2004;22:233-41.

20. Pannu J, Nakerakanti S, Smith E, ten Dijke P, Trojanowska M. Transforming growth factor-beta receptor type I-dependent fibrogenic gene program is mediated via activation of Smad 1 and ERK1/2 pathways. J Biol Chem. 2007;282:10405-13. 
TABLE E1. Sequences of Smad1 siRNA and scrambled siRNA

\begin{tabular}{ll}
\hline & \multicolumn{1}{c}{ Knockdown siRNA pool } \\
\hline Smad1 & Sense 5'-GCUCUAUUGUCUACUAUGAUU-3' \\
& Antisense 5'-PUCAUAGUAGACAAUAGAGCUU-3' \\
& Sense 5'-GGCGGUUGCUUAUGAGGAAUU-3' \\
& Antisense 5'-PUUCCUCAUAAGCAACCGCCUU-3' \\
& Sense 5'-CAACAAUCGUGUGGGUGAAUU-3' \\
& Antisense 5'-PUUCACCCACACGAUUGUUGUU-3' \\
& Sense 5'-CAAAUGGGUUCACCUCAUAUU-3' \\
& Antisense 5'-PUAUGAGGUGAACCCAUUUGUU-3' \\
& Sense 5'-UAGCGACUAAACACAUCAAUU-3' \\
Scrambled & Antisense 5'-PUUGAUGUGUUUAGUCGCUAUU-3' \\
siRNA &
\end{tabular}

siRNA, Small interfering RNA. 